\title{
Beklenti Maksimizasyonu ile Genişletilmiş Hedef İzleme
}

\author{
${ }^{1}$ Emre ÖZKAN* \\ ${ }^{1}$ Orta Doğu Teknik Üniversitesi, Mühendislik Fakültesi, Elektrik Elektronik Mühendisliği \\ Bölümü, Ankara
}

Geliş tarihi: 27.01.2019

Kabul tarihi: 27.03.2019

\section{$\ddot{\mathbf{O} z}$}

Bu çalışmada genişletilmiş hedef izleme (GHI) problemi ele alınmıştır. GHİ problemi klasik hedef izleme probleminden farklı olarak, bir hedefin tek bir anda birden fazla ölçüme sebep olması durumunu inceler. $\mathrm{Bu}$ varsayım altında toplanan ölçümlerden, hedefin hem kinematik bilgileri hem de şekli kestirilir. Literatürde bu problemi çözmeye yönelik yaklaşık çözümlü algoritmalar vardır. Ancak bu çalışmaların pek çoğu teorik alt yapısı zayıf olan buluşsal çözüm önerileri içerir. Bu çalışmada yüzeyi birden çok elips ile gösterilebilen bir hedefi takip eden ve hedefin şeklini öğrenebilen beklenti maksimizasyonu (BM) temelli yeni bir yöntem geliştirilmiştir. GHI problemi stokastik durum uzay modellerinde parametre kestirimi problemi haline getirilmiş ve parçacık filtresi kullanarak kestirim yapılmıştır. Simülasyonlarda çoklu elipsten oluşan ve bilinmeyen şekle sahip bir genişletilmiş hedef isabetle takip edilmiş ve hedefin şekli başarıyla kestirilmiştir.

Anahtar Kelimeler: Parçacık filtresi, Beklenti maksimizasyonu, Genişletilmiş hedef izleme

\section{Expectation Maximization Based Extended Target Tracking}

\begin{abstract}
In this study, extended target tracking (ETT) problem is considered. The ETT problem, unlike the classical target tracking problem, makes the assumption that a target generates more than one measurement at a time. Both the kinematic state and the shape of targets are estimated from the measurements collected under this assumption. In the literature, there are approximate solution algorithms to solve this problem. However, many of these studies include heuristic approximations. In this paper, we develop a new expectation maximization (EM) based method, which can track and learn the shape of an extended target whose extent can be represented by multiple ellipses. For this purpose, we cast the ETT problem as a parameter estimation problem in stochastic state space models, and perform estimation using particle filters. In the simulations, an extended target with unknown shape consisting of multiple ellipses is tracked accurately, and the shape of the target is estimated successfully.
\end{abstract}

Keywords: Particle filter, Expectation maximization, Extended target tracking

"Sorumlu Yazar (Corresponding Author): Emre ÖZKAN, emreo@metu.edu.tr 


\section{GíRiș}

Hedef izleme, belli bir alanda hareket eden nesneleri, sensörlerle elde edilen gürültülü ölçümlere bakarak takip edebilme problemidir. $\mathrm{Bu}$ ölçümler arasında çoğu zaman yanlış alarmlar da mevcuttur. Geleneksel tekniklerde hedefler, ölçümleri merkezi etrafinda toplanmış noktasal nesneler olarak modellenir ve tek bir anda sadece bir ölçüm yarattıkları varsayımı yapılır [1]. Pek çok radar uygulamasında bu varsayım vardır. Ancak gelişen sensör ve hesaplama teknolojileri sayesinde hedeflerle ilgili tek ölçüm yerine farklı yansıma noktalarından toplanmış çoklu ölçümler elde edilebilmektedir. Tek bir anda aynı hedefe ait birden fazla ölçümü işleyebilen sistemlere genişletilmiş hedef izleme (GHI) sistemleri denir [2]. GHİ için literatürde çeşitli yaklaşımlar önerilmiştir. Bu çalışmalar en genel haliyle tek bir nesneyi basit bir geometrik şekille modellemeye dayanır. Bu geometrik şekil dikdörtgen, elips ya da daire olabilir. Eliptik obje modellemesi için son yıllarda yaygın olarak kullanılan yöntem, rastlantısal matris (RM) metodudur [3-4]. RM metodunda bir hedefe ait ölçümlerin kovaryans matrisi bilinmeyen bir Gauss dağılımından geldiği var sayılır ve bu dağılımın kovaryans matrisi kestirilmeye çalışılır. Bu yaklaşımın doğal sonucu olarak, bu yöntemle izlenen hedeflerin yüzeyleri tek bir elips ile gösterilir. $\mathrm{Bu}$ yöntemler sınırlandırıcı varsayımlar altında [3] ya da sezgisel hesaplamalarla [4] çalışmakta ve hedefe dair detayları, eliptik yaklaşıklama ile kaybetmektedirler.

$\mathrm{Bu}$ çalışmada birden fazla ölçüm yaratan hedeflerin çoklu elipslerle modellenmesi ve böylece hedefe ait farklı yansıma kaynaklarının daha isabetli takip edilebilmesi amaçlanmıştır. $\mathrm{Bu}$ amacı gerçekleştirebilmek için, istatistik teorisindeki bazı tekniklerin en yeni halleri kullanılmıştır. Çözüm önerimiz maksimum olabilirlik kestirimi üzerine kuruludur ve bu kestirimi bulabilmek için beklenti maksimizasyonu (BM) algoritması kullanılmıştır.

BM algoritması maksimum olabilirlik kestiriminde oldukça yaygın olarak kullanılan bir metottur [5].
İstatistik, biyoloji, sinyal işleme, yapay zeka ile öğrenme gibi pek çok alanda numerik optimizasyon metotlar1 yerine tercih edilir [6-8]. BM algoritmasının bir dezavantaj1, bu yöntemin ancak tüm veri toplandiktan sonra uygulanabilmesidir. Genel durum uzay modelleri parametre kestirimi için BM kullanımı [9] numaralı referansta özetlenmiştir. Pek çok gerçek zamanlı uygulamada kestirim probleminin anında çözülmesi gerekir. Son yıllarda BM algoritmasını bu tür problemlerde kullanabilmek için anında veri işleyen versiyonları önerilmiştir [10-11]. Bu yöntemlerde veriler toplandıkça kullanılır ve tüm veriyi saklamaya gerek yoktur. $\mathrm{Bu}$ yöntemin doğrusal olmayan durum uzay modellerinde parametre ve durum vektörünün birlikte kestirimi için uyarlanmış hali [11] numaralı referansta anlatılmıştır.

BM algoritmasını GHİ problemine uygulamak için uygun çıkarım tekniği kullanılmalıdır. Bu çalışmada bu amaç için parçacık filtresi kullanılmıştır [12].

Makalenin geri kalanı aşağıda anlatıldı ̆̆ı gibi düzenlenmiştir. Bölüm 2'de BM ve GHİ probleminin temel eşitlikleri verilmiştir. Yine bu bölümde, üstel dağılım ailesi ve GHİ de kullanılan eşleme değişkenleri tanıtılmıştır. 3. bölümde, durum uzay modellerinde anlık ve toplu BM algoritmalarının parçacık filtresiyle gerçeklenmesi ve anlık BM metodu ile GHİ probleminin çözümü anlatılmıştır. 4. bölümde benzetim sonuçları, 5 . bölümde ise tartışma ve sonuç verilmiştir.

\section{TEMEL DENKLEMLER}

\subsection{Beklenti Maksimizasyonu}

BM algoritması bilinmeyen parametrelerin maksimum olabilirlik kestirimini bulmak için kullanılır. Algoritmanın detaylı bir incelemesi [6] numaralı referansta verilmiştir. Pek çok problemde olabilirlik fonksiyonunu parametrelere göre maksimize etmek güçtür. BM algoritması bu güç problemi yeni değişkenler tanımlayarak, daha kolay çözülebilen, birbiriyle ilintili iki küçük 
probleme indirger ve bu problemleri iterasyonlar yaparak çözer. BM algoritması bir deney sonucu toplanmış verinin tamamını, veriler toplandıktan sonra işleyebilen bir algoritmadır. Ancak, son yıllarda verinin tamamının toplanmasına, saklanmasına ihtiyaç duymayan; veri toplanırken de kestirim yapabilen BM metotları önerilmiştir $[10,11]$. Biz bu yöntemleri anlık (online) BM; tüm veri toplandıktan sonra çalışan BM metotlarını da toplu (batch) BM olarak adlandıracağız.

\subsubsection{Toplu BM}

Toplu BM algoritmasını anlatmadan önce bir deney sonucu elde ettiğimizi var saydığımız ölçümleri tanımlayalım (Eşitlik 1),

$$
\mathrm{Y}=\left\{\mathrm{y}_{1}, \ldots, \mathrm{y}_{\mathrm{n}}\right\} \text {. }
$$

Bilinmeyen parametreleri $\theta$ ile gösterirsek, bulmak istediğimiz $\theta$ kestirimi Eşitlik 2'de verilmiştir:

$\hat{\theta}^{\mathrm{BM}}=\underset{\theta}{\operatorname{argmax}} \mathrm{p}_{\theta}\left(\mathrm{y}_{1}, \ldots, \mathrm{y}_{\mathrm{n}}\right)$

Bir başka deyişle amacımız ölçümlere en çok "uyan" maksimum olabilirlikli parametre değerlerini bulmaktır. Benzer bir şekilde, numerik kolaylık sağlamasından dolayı maksimum olabilirlik fonksiyonunun logaritması da maksimize edilebilir. Pek çok sistemde, gözlenebilen ölçümler, gözlemlenemeyen değişkenler ile olan ilişkileriyle beraber modellenir. $\mathrm{Bu}$ gizli değişkenleri aşağıdaki gibi tanımlayalım,

$\mathrm{X}=\left\{\mathrm{x}_{1}, \ldots, \mathrm{x}_{\mathrm{n}}\right\}$

$\mathrm{Bu}$ değişkenler, gözlemlenemeyen sistem yapısını ve bu yapının ölçümlerle olan ilişkisini açıklar. $p_{\theta}(Y)$ fonksiyonu maksimize edilirken, daha kolay irdelenebilen $p_{\theta}(Y, X)$ ortak olasılık fonksiyonu kullanılabilir [6]. BM algoritması bu ortak olasılık yoğunluk fonksiyonunu kullanarak bilinmeyen parametrenin maksimum olabilirlik kestirimini iterasyonlarla bulmaya çalışır. Algoritmanın $m$ 'inci iterasyonunda Eşitlik 3 ve 4'te verilen işlemler yapilır [6].

\section{B-Adımı:}

$\mathrm{Q}\left(\theta \mid \theta_{\mathrm{m}-1}\right)=\mathrm{E}_{\mathrm{p}_{\theta_{\mathrm{m}-1}}(\mathrm{X} \mid \mathrm{Y})}\left\{\log \mathrm{p}_{\theta}(\mathrm{X}, \mathrm{Y})\right\}$

\section{M-Adımı:}

$\theta_{\mathrm{m}}=\underset{\theta}{\operatorname{argmax}} \mathrm{Q}\left(\theta \mid \theta_{\mathrm{m}-1}\right)$

Beklenti, yani B-adımında, tüm veri logolabilirliğinin $\log p_{\theta}(X, Y)$, gizli değişkenlerin koşullu olasılık yoğunluk fonksiyonuna göre $\left(p_{\theta_{m-1}}(X \mid Y)\right)$ beklentisi hesaplanır. Koşullu olasilik fonksiyonu parametrelerin son tahmini olan ve bir önceki iterasyonda hesaplanan $\theta_{m-1}$ parametre değerleri kullanılarak hesaplanır.

Maksimizasyon, yani M-adımında ise, B-adımında hesaplanan beklenti fonksiyonu $\theta$ 'ya göre maksimize edilir [6].

BM algoritmasının her iterasyonu sonunda yeni bir parametre kestirimi bulunur. Bulunan yeni kestirim, bir önceki değerine göre, (2) numaralı eşitlikte maksimize edilen ölçümlerin olabilirlik fonksiyonunu artırır ya da eşit kılar [6], [13].

(3) ve (4) numaralı eşitlikler BM algoritmasının temel mekanizmasını tanımlar. Ardışık olarak B ve $\mathrm{M}$ adımlarını tekrar eden algoritma, bir süre sonra marjinal olabilirlik fonksiyonunun ekstremum noktalarından birine yakınsar [6].

$B$ ve $M$ adımlarının daha da kolay çözülebileceği özel durumlar mevcuttur. $\mathrm{Bu}$ makalede bu adımların analitik ifadelerle çözülebileceği bir çerçeve tanımlayıp, genişletilmiş hedef izleme problemini çözmeyi amaçlayacağız. Aşağıdaki koşullar sağlandığı takdirde $\mathrm{B}$ ve $\mathrm{M}$ adımlarının kolay çözümü mümkün olmaktadır.

1. Tüm veri olabilirliği $p_{\theta}(X, Y)$ 'nin üstel dağılım ailesinden olması.

2. Tüm veri olabilirliğinin maksimum değerinin analitik ifade edilebilmesi.

Birinci özellik B-adımında, ikinci özellik ise M-adımında, $Q($.$) fonksiyonunun maksimumunu$ sağlayan $\theta$ değerini bulmakta kolaylık sağlar.

\section{2. Üstel Dağılım Ailesi}


Olasılık dağılım fonksiyonlarının bir sınıfına tekabül eden bu aileye ait olan dağılım fonksiyonları Eşitlik 5'te verilen parametrizasyonla gösterilebilirler [13].

$\mathrm{p}_{\theta}(\mathrm{x})=\mathrm{c} \exp (\langle\psi(\theta), \mathrm{s}(\mathrm{x})\rangle-\mathrm{A}(\theta))$

Bu ifadede $c, \theta$ 'ya bağlı olmayan bir fonksiyon; $\langle.,$.$\rangle içsel çarpım; \mathrm{s}(\mathrm{x})$ tüm data yeterli istatistikleri, $\psi($.$) ve \mathrm{A}($.$) ise \theta$ 'nın birer fonksiyonlarıdır.

Bilinen pek çok dağılım, üstel dağılım ailesi mensubudur. $\mathrm{Bu}$ dağılımlara örnek olarak Gauss, Poisson, Dirichlet ve Wishart dağılımları gösterilebilir. Üstel dağılımlar, sağladıkları pek çok cebirsel kolaylıktan dolayı istatistik teorisinde sıkça kullanılır.

\subsection{Genişletilmiş Hedef İzleme}

GHİ probleminde amaç, noktasal olmayan ve belirli bir yüzeyden ölçümler üreten hedeflerin dinamik durum vektörlerini ve ölçüm yaratan yüzeylerini kestirmektir. Basit bir elipsin ölçüm yüzeyini modellemede yetersiz kaldığı durumlar için bu çalışmada bir örneği Şekil 1'de gösterilen çoklu elips modeli önerilmiştir.

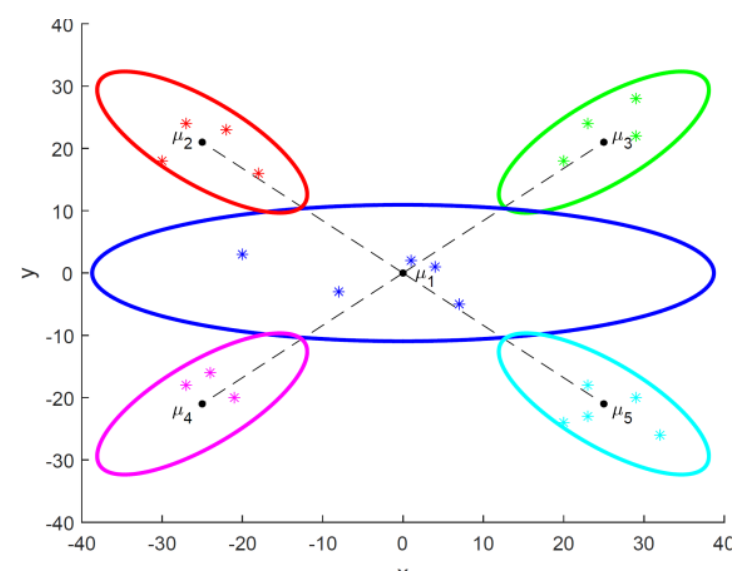

Şekil 1. Çoklu elips GHİ modeli. Beş alt-objeden oluşan bu örnek hedefte alt-objelere ait olan ölçümler '*' ile gösterilmiştir
Bu modelde alt-obje olarak nitelendirilen elipslerin her biri, birer yar1-kesin artı rastlantısal matris ile tanımlanır. $K$ adet alt-obje olduğu varsayımı altında bu matrisleri $\left\{\mathrm{X}_{\mathrm{k}}^{1}\right\}_{\mathrm{l}=1}^{\mathrm{K}}$ ile gösterelim. Aynı elipslerin hedef merkezine $\left(x_{k}^{c}\right)$ olan uzaklıklarını $\left\{\mu_{\mathrm{k}}^{1}\right\}_{1=1}^{\mathrm{K}}$ ile gösterelim. Bu tanımlar altında, herhangi bir $k$ anında bu hedeften gelen $m_{k}$ adet ölçümün her biri $\left(y_{k}^{j}\right)$ Eşitlik 6'da verilen ölçüm eşitliğini sağlar.

$$
\begin{aligned}
& \mathrm{p}\left(\mathrm{y}_{\mathrm{k}}^{\mathrm{j}} \mid \mathrm{x}_{\mathrm{k}}^{\mathrm{c}}, \mathrm{X}_{\mathrm{k}}^{1: \mathrm{K}}, \mu_{\mathrm{k}}^{1: \mathrm{K}}\right) \\
&=\sum_{\mathrm{l}=1}^{\mathrm{K}} \pi^{1} \mathrm{~N}\left(\mathrm{y}_{\mathrm{k}}^{\mathrm{j}} ; \mathrm{x}_{\mathrm{k}}^{\mathrm{c}}+\mu_{\mathrm{k}}^{1}, \mathrm{X}_{\mathrm{k}}^{1}\right)
\end{aligned}
$$

Gauss karışımına karşılık gelen bu eşitlikte, $\mathrm{N}(\mathrm{a} ; \mathrm{b}, \Sigma) \quad a$ rastlantısal değişkeni için $b$ ortalama değerli, $\Sigma$ kovaryanslı Gauss dağılımını; $\pi_{l}$ değişkeni ise [0,1] aralığında değer alan karışım ağırlıklarını gösterir. Bilinmeyen parametreleri $\theta=\left\{\pi^{1: \mathrm{K}}, \mu_{\mathrm{k}}^{1: \mathrm{K}}, \mathrm{X}_{\mathrm{k}}^{1: \mathrm{K}}\right\} \quad$ olarak tanımladığımızda amacımız durum vektörü $x_{k}^{c}$ ve $\theta^{\prime}$ yı beraber kestirebilmektir.

$\mathrm{Bu}$ ortak kestirim problemini çözebilmek oldukça zordur. Zorluk sebeplerinin başında, herhangi bir anda elde edilen bir ölçümün $\left(\mathrm{y}_{\mathrm{k}}^{\mathrm{j}}\right)^{\prime}$ 'nin hangi elipse ait olduğunun bilinmemesi gelir. Bu problem, her gelen ölçümle ve zamanla karmaşıklığı artan, kombinatorik bir problemdir.

Bir sonraki bölümde, hedef izleme literatüründe eşleme problemi olarak da bilinen bu problemi çözebilmek için önerilen eşleme değişkenleri anlatılacaktır.

\subsubsection{Eşleme Değişkenleri}

Gelen ölçümlerle alt-objeler arasında eşleştirme yapabilmek için $k$ anında gelen $\mathrm{m}_{\mathrm{k}}$ adet ölçümün her biri için bir eşleme değişkeni tanımlayalım. j’inci ölçüm $\mathrm{y}_{\mathrm{k}}^{\mathrm{j}} \mathrm{için}$ bu değişkeni $\mathrm{r}_{\mathrm{k}}^{\mathrm{j}}$ ile gösterelim. $K$ adet alt-objenin olduğu durumda, $\mathrm{r}_{\mathrm{k}}^{\mathrm{j}}$ değişkenleri 1 ile $K$ arasında tam sayı değerler alırlar. Gelen 
ölçümün etiketi olarak nitelendirilebilecek bu değişkenler, ölçümün hangi alt-objeye ait olduğunu gösterirler.

$\mathrm{Bu}$ varsayım altında bir hedefe ait durum-uzay modeli Eşitlik 7 ve 8'deki gibi yazılabilir.

$\mathrm{x}_{\mathrm{k}}=\mathrm{f}\left(\mathrm{x}_{\mathrm{k}-1}\right)+\mathrm{e}_{\mathrm{k}}$

$y_{k}^{j}=h\left(x_{k}\right)+w_{k}\left(r_{k}^{j}\right)$

$\mathrm{Bu}$ eşitliklerde, $x_{k}$ hedefe ait durum vektörünü; $f($.$) durum vektörünün dinamiğini; h($.) durum vektörünü ölçümlerle ilişkilendiren fonksiyonu; $e_{k} \sim N\left(e_{k} ; 0, Q\right) \quad$ ve $w_{k}\left(r_{k}^{j}\right) \sim N\left(w_{k} ; \mu_{k}^{r^{j}}, X_{k}^{j^{j}}\right)$, Gauss dağılımına sahip bağımsız beyaz rastlantısal değişkenleri, Q süreç gürültüsü kovaryans matrisini gösterir. Bir ölçümün l'inci alt-objeye ait olma öncül olasılığını Eşitlik 9'daki gibi tanımlarsak,

$$
P\left(r_{k}^{j}=l\right)=\pi^{l}, \quad l \in\{1, . ., K\}
$$

ve $h($.$) fonksiyonunu h\left(x_{k}\right)=x_{k}^{c}$ olmak üzere seçersek, elde edilen ölçüm eşitliği Eşitlik 6'da verilen $\left\{\pi^{l}\right\}_{l=1}^{K}$ ağırlıklarıyla ağırlıklandırılmış, Gauss karışımına karşılık gelir.

Eşleme değişkenlerin tanımıyla beraber GHİ problem için kullanacağımız durum vektörünü kesikli ve sürekli değişkenlerin bir kombinasyonu olarak $z_{k} \triangleq\left[x_{k}^{T} r_{k}^{T}\right]^{T}$ tanımlayabiliriz. Bir sonraki kısımda bu değişken ve bilinmeyenler üzerinden parçacık filtresi kullanarak Anlık BM algoritmasının nasıl gerçeklenebileceğini göreceğiz.

\section{METOT}

Bir önceki bölümde tanımlanan durum vektörü $z_{k}$ 'yı gizli değişken olarak kullanarak, BM algoritmasının B-adımında kullanılan ortak olasılık yoğunluk fonksiyonunu Eşitlik 10'daki gibi yazabiliriz,

$\mathrm{p}_{\theta}\left(\mathrm{z}_{0: \mathrm{T}}, \mathrm{y}_{0: \mathrm{T}}\right)=\prod_{\mathrm{k}=0}^{\mathrm{T}} \mathrm{p}_{\theta}\left(\mathrm{z}_{\mathrm{k}}, \mathrm{y}_{\mathrm{k}} \mid \mathrm{z}_{\mathrm{k}-1}\right)$.
$\mathrm{Bu}$ ifadenin sağ tarafinda yer alan her bir çarpan Eşitlik 11'deki gibi yazılabilir.

$$
\mathrm{p}_{\theta}\left(\mathrm{z}_{\mathrm{k}}, \mathrm{y}_{\mathrm{k}} \mid \mathrm{z}_{\mathrm{k}-1}\right)=\mathrm{p}_{\theta}\left(\mathrm{y}_{\mathrm{k}} \mid \mathrm{z}_{\mathrm{k}}\right) \mathrm{p}_{\theta}\left(\mathrm{z}_{\mathrm{k}} \mid \mathrm{z}_{\mathrm{k}-1}\right)
$$

$\mathrm{Bu}$ ifade yine üstel dağılım ailesi mensubu olduğunda aşağıdaki gibi ifade edilebilir.

$p_{\theta}\left(z_{k}, y_{k} \mid z_{k-1}\right)=C \exp \left(\psi(\theta), s\left(z_{k}, z_{k-1}, y_{k}\right)-A(\theta)\right)$

Bu eşitlikte $C, \theta$ 'dan bağımsız bir sabiti; $\langle.,$.$\rangle içsel$ çarpımı; $\mathrm{s}\left(\mathrm{z}_{\mathrm{k}}, \mathrm{z}_{\mathrm{k}-1}, \mathrm{y}_{\mathrm{k}}\right)$ tüm data yeterli istatistiklerini; $\psi($.) ve $\mathrm{A}($.) ise $\theta$ 'nın bir fonksiyonunu gösterir. $\mathrm{Bu}$ varsayımlara ek olarak B-Adımının maksimum değerinin analitik olarak ifade edilebildiği durumlarda, Eşitlik 3 ve 4'te verilen BM algoritması iterasyonları Eşitlik 13 ve 14 'teki forma dönüşür.

\section{B-Adımı:}

$\mathrm{S}_{\mathrm{m}}=\frac{1}{\mathrm{~T}} \mathrm{E}_{\mathrm{p}_{\theta_{\mathrm{m}-1}}\left(\mathrm{z}_{0: \mathrm{T}} \mid \mathrm{y}_{0: \mathrm{T}}\right)}\left\{\sum_{\mathrm{k}=1}^{\mathrm{T}} \mathrm{s}\left(\mathrm{z}_{\mathrm{k}}, \mathrm{z}_{\mathrm{k}-1}, \mathrm{y}_{\mathrm{k}}\right)\right\}$

\section{M-Adımı:}

$\theta_{\mathrm{m}}=\Phi\left(\mathrm{S}_{\mathrm{m}}\right)=\underset{\theta}{\operatorname{argmax}}\left[\left\langle\psi(\theta), \mathrm{S}_{\mathrm{m}}\right\rangle-\mathrm{A}(\theta)\right]$

(14) numaralı eşitlikte $\Phi($.$) fonksiyonu, S_{m}$ yeterli istatistiklerini kullanarak, M-adımının maksimum değerini sağlayan $\theta$ parametresini kapalı form ifade edebilen fonksiyondur.

\subsection{Anlık BM}

Toplu BM algoritması tüm ölçümler $\quad y_{0: T}$ toplandıktan sonra bu ölçümlerin tamamını kullanarak gizli değişkenler $z_{0: T}$ ve $\theta^{\prime}$ 'y kestirmeye çalışır. Anlık BM algoritmasında ise bu kestirim, gelen her ölçümle beraber, verilerin toplandığı sırada yapılabilir. Bu özellik, anlık kestirim gereksinimi olan gerçek zamanlı uygulamalarda önem taşır. Toplu BM algoritması uygun modifikasyonlarla anlık BM algoritmasına çevrilebilir [10,11]. Anlık BM'da herhangi bir k anında sadece o ana kadar toplanan ölçümler $\left(y_{0: k}\right)$ kullanılır. Her bir zaman aralığında BM 
iterasyonları sadece bir kez yapılır. Eşitlik 15'te yardımcı fonksiyon kullanarak Anlı BM algoritması gerçeklenebilir [11].

$\rho_{\mathrm{k}}\left(\mathrm{z}_{0: \mathrm{k}}\right)=\frac{1}{\mathrm{k}} \sum_{\mathrm{t}=1}^{\mathrm{k}} \mathrm{s}\left(\mathrm{z}_{\mathrm{t}}, \mathrm{z}_{\mathrm{t}-1}, \mathrm{y}_{\mathrm{t}}\right)$

Tanımlanan bu yardımcı fonksiyonla, yeterli istatistikler Eşitlik 16'daki integral ile hesaplanır.

$$
S_{k}=\int \rho_{k}\left(z_{0: k}\right) p_{\theta_{k}}\left(z_{0: k} \mid y_{0: k}\right) d z_{0: k}
$$

Yardımcı fonksiyon $\rho_{k}\left(z_{0: k}\right)$ özyineli olarak hesaplanabilir. (Eşitlik 17)

$$
\rho_{k}\left(z_{0: k}\right)=\gamma_{k} s\left(z_{k}, z_{k-1}, y_{k}\right)+\left(1-\gamma_{k}\right) \rho_{k-1}\left(z_{0: k-1}\right)
$$

Ağırlıklandırılmış toplam şeklinde ilerleyen bu özyineli eşitlikler, zamana göre değişen adım aralıklarıyla stokastik yakınsama varsayımlarına uygun hale getirilmiştir. Ağırlıklandırmada kullanılan $\left\{\gamma_{\mathrm{k}}\right\}_{\mathrm{k} \geq 1}$ adım aralığı $\sum_{\mathrm{k} \geq 1} \gamma_{\mathrm{k}} \rightarrow \infty$, ve $\sum_{\mathrm{k} \geq 1} \gamma_{\mathrm{k}}^{2}<\infty$ şartlarını sağladığı takdirde yakınsama sağlanır [11].

$\mathrm{Bu}$ uyarlama sonucu elde edilen anlık BM algoritması Eşitlik 18a, 18b ve 18c'deki gibi özetlenmiştir.

\section{B-Adımı:}

$$
\begin{aligned}
& p_{\theta_{k-1}}\left(z_{0: k} \mid y_{0: k}\right)= \\
& \frac{p_{\theta_{k-1}}\left(z_{k}, y_{k} \mid z_{k-1}\right) p_{\theta_{k-1}}\left(z_{0: k-1} \mid y_{0: k-1}\right)}{\int p_{\theta_{k-1}}\left(z_{k}, y_{k} \mid z_{k-1}\right) p_{\theta_{k-1}}\left(z_{0: k-1} \mid y_{0: k-1}\right) d z_{0: k}} \\
& \rho_{k}\left(z_{0: k}\right)=\gamma_{k} s\left(z_{k}, z_{k-1}, y_{k}\right)+\left(1-\gamma_{k}\right) \rho\left(z_{0: k-1}\right) \\
& S_{k}=\int \rho_{k}\left(z_{0: k}\right) p_{\theta_{k}}\left(z_{0: k} \mid y_{0: k}\right) d z_{0: k}
\end{aligned}
$$

\section{M-Adımı:}

(Eşitlik 18d)

$\theta_{k}=\Phi\left(S_{k}\right)$

\subsection{Anlık BM ve Parçacık Filtresi}

Yukarıda verilen Anlık BM algoritması pek çok problem için kapalı form eşitliklerle ifade edilemez. $\mathrm{Bu}$ tür durumlarda, ilgili olasılık yoğunluk fonksiyonlarının parçacık filtresi ile yaklaşıklanması anlık BM algoritmasının gerçeklenmesini mümkün kılar. Parçacık filtresi doğrusal olmayan durum uzay modellerinde sonsal dağılımı bulmak için kullanılır. Bu sonsal dağılım, önceden belirlenmiş sayıda parçacık ve ağırlıklarıyla Eşitlik 19'daki gibi yaklaşıklanır.

$$
p\left(z_{0: k} \mid y_{0: k}\right) \approx \sum_{i=1}^{N} w_{k}^{(i)} \delta\left(z_{0: k}-z_{0: k}^{(i)}\right) .
$$

$\mathrm{Bu}$ yaklaşıklamada kullanılan ağırlıkların $\left(w_{k}^{(i)}\right)$ toplamı 1'dir, $\sum_{i=1}^{N} w_{k}^{(i)}=1 . \quad \mathrm{Bu}$ ağırlıklar, özyineli bir şekilde Eşitlik 20'deki gibi hesaplanır.

$w_{k}^{(i)}=w_{k-1}^{(i)} \frac{p\left(y_{k} \mid z_{k}^{(i)}\right) p\left(z_{k}^{(i)} \mid z_{k-1}^{(i)}\right)}{q\left(z_{k}^{(i)} \mid z_{k-1}^{(i)}, y_{k}\right)}$

$\mathrm{Bu}$ eşitlikte $\mathrm{q}\left(\mathrm{z}_{\mathrm{k}}^{(\mathrm{i})} \mid \mathrm{z}_{\mathrm{k}-1}^{(\mathrm{i})}, \mathrm{y}_{\mathrm{k}}\right)$ önem fonksiyonunu gösterir. $\mathrm{Bu}$ yaklaşımla $\rho_{\mathrm{k}}\left(\mathrm{z}_{0: \mathrm{k}}\right)$ fonksiyonu da yaklaşıklanabilir. $\mathrm{Bu}$ durumda Anlık BM algoritmasının B-Adımı için gereken $S_{k}$ değişkeninin kestirimi Eşitlik 21'deki gibi hesaplanır.

$\widehat{\mathrm{S}_{\mathrm{k}}}=\sum_{\mathrm{i}=1}^{\mathrm{N}} \mathrm{w}_{\mathrm{k}}^{(\mathrm{i})} \rho_{\mathrm{k}}^{(\mathrm{i})}\left(\mathrm{z}_{0: \mathrm{k}}^{(\mathrm{i})}\right)$

Son olarak, kestirilen $\widehat{S_{k}}$ değişkeni, yeni parametre değerlerini bulmak üzere, maksimizasyon fonksiyonuna verilir. (Eşitlik 22)

$\theta_{\mathrm{k}}=\Phi\left(\widehat{\mathrm{S}_{\mathrm{k}}}\right)$

Algoritma-1'de anlık BM için kullanılan, ve öncül dağılımdan örneklem alan parçacık filtresinin akış kodu verilmiştir. Bu kod simülasyon senaryosunda kullanılmıştır.

Algoritma-1: Anlık BM Parçacık Filtresi 


\section{Başlangıç:}

Her bir parçackk için $\mathrm{i}=1, \ldots, \mathrm{N}$

- $x_{0}^{i} \sim p\left(x_{0}\right)$ 'leri örnekle

- Başlangıç ağırlıklarını belirle $w_{0}^{i}=N^{-1}$

\section{Güncellemeler:}

Her $\mathrm{k}=1,2, \ldots$, için,

- Her bir parçacık için $\mathrm{i}=1, \ldots, N$

Örnekleme:

$\mathrm{r}_{\mathrm{k}}^{\mathrm{i}} \sim \pi(),. \mathrm{x}_{\mathrm{k} \mid \mathrm{k}-1}^{\mathrm{i}} \sim \mathrm{p}\left(\mathrm{x}_{\mathrm{k}} \mid \mathrm{x}_{\mathrm{k}-1}^{\mathrm{i}}, \mathrm{y}_{\mathrm{k}-1}\right)-$

- Ağırlıkları güncelle: $\widetilde{\mathrm{w}}_{\mathrm{k}}^{\mathrm{i}}=\prod_{\mathrm{j}=1}^{\mathrm{m}_{\mathrm{k}}} \mathrm{p}\left(\mathrm{y}_{\mathrm{k}}^{\mathrm{j}} \mid \mathrm{r}_{\mathrm{k}}^{\mathrm{i}, \mathrm{j}}, \mathrm{x}_{\mathrm{k}}^{\mathrm{i}}\right) \mathrm{w}_{\mathrm{k}-1}^{\mathrm{i}}$,

- Ağırlık toplamlarını 1'e eşitle $\mathrm{w}_{\mathrm{k}}^{\mathrm{i}}=\frac{\widetilde{\mathrm{w}}_{\mathrm{k}}^{\mathrm{i}}}{\sum_{\mathrm{i}=1}^{\mathrm{N}} \widetilde{\mathrm{w}}_{\mathrm{k}}^{\mathrm{i}}}$,

- Her bir parçacık için $\mathrm{i}=1, \ldots, N$

Yardımcı fonksiyonu $\left(\rho_{k+1}^{i}\right)$ güncelle

$\rho_{\mathrm{k}}^{\mathrm{i}}\left(\mathrm{z}_{0: \mathrm{k}}^{\mathrm{i}}\right)=\gamma_{\mathrm{k}} \mathrm{s}\left(\mathrm{z}_{\mathrm{k}}^{\mathrm{i}}, \mathrm{z}_{\mathrm{k}-1}^{\mathrm{i}}, \mathrm{y}_{\mathrm{k}}\right)+\left(1-\gamma_{\mathrm{k}}\right) \rho_{\mathrm{k}-1}^{\mathrm{i}}\left(\mathrm{z}_{0: \mathrm{k}-1}^{\mathrm{i}}\right)$

- Parametre Kestirimi yap

$S_{k}=\sum_{i=1}^{N} w_{k}^{(i)} \rho_{k}^{(i)}, \theta_{k}=\Phi\left(S_{k}\right)$

- Parçacıkları yeniden örnekle

\subsection{GHI Probleminin Anlık BM ve Parçacık Filtresi ile Çözümü}

(7) ve (8) numaralı denklerle ifade edilen genişletilmiş hedef modelinde bilinmeyen parametreler, her bir elipsi temsil eden ortalama değer vektörleri ve kovaryans matrisleridir,

$\theta=\left\{\mu_{\mathrm{k}}^{1}, \mathrm{X}_{\mathrm{k}}^{1}, \mu_{\mathrm{k}}^{2}, \mathrm{X}_{\mathrm{k}}^{2}, \ldots, \mu_{\mathrm{k}}^{\mathrm{K}}, \mathrm{X}_{\mathrm{k}}^{\mathrm{K}}\right\}$

Bir ölçümün herhangi bir alt-objeye ait olma öncül olasılığının bilinmediği durumlarda, bu değişken de bilinmeyen parametrelere dahil edilebilir, $\theta=\left\{\mu_{\mathrm{k}}^{1}, \mathrm{X}_{\mathrm{k}}^{1}, \mu_{\mathrm{k}}^{2}, \mathrm{X}_{\mathrm{k}}^{2}, \ldots, \mu_{\mathrm{k}}^{\mathrm{K}}, \mathrm{X}_{\mathrm{k}}^{\mathrm{K}}, \pi_{1: \mathrm{K}}\right\}$

Alogritma-1 de anlatılmış olan metodu uygulayabilmek için bilinmeyenlerin yanı sıra, yeterli istatistikler $s\left(z_{k}, z_{k-1}, y_{k}\right)$ ve bilinmeyen parametrelerle istatistikleri ilişkilendiren maksimizasyon fonksiyonunun $\Phi($.$) tanımlanması$ gereklidir. Maksimizasyon fonksiyonu $\Phi($.$) ,$ M-adımında Eşitlik 22'deki gibi hesaplanır. Verilen modelde bilinmeyen parametreler yeterli istatistiklerden Eşitlik 23a-23c kullanılarak hesaplanır.

$\hat{\mu}_{k}^{j}=\Phi\left(\hat{S}_{k}^{\mu}, \hat{S}_{k}^{\pi}\right)=\frac{\hat{S}_{k}^{\mu}(j)}{\hat{S}_{k}^{\pi}(j)}$

$\hat{X}_{k}^{j}=\Phi\left(\hat{S}_{k}^{X}, \hat{S}_{k}^{\pi}\right)=\frac{\hat{S}_{k}^{X}(j)}{\hat{S}_{k}^{\pi}(j)}-\hat{\mu}_{k}^{j}\left(\hat{\mu}_{k}^{j}\right)^{T}$

$\hat{\pi}_{k}^{j}=\Phi\left(\hat{S}_{k}^{\pi}\right)=\frac{\hat{S}_{k}^{\pi}(j)}{\sum_{i=1}^{K} \hat{S}_{k}^{\pi}(i)}$

$\hat{S}_{k}^{X}, \hat{S}_{k}^{\pi}, \hat{S}_{k}^{\mu}$ değişkenleri Eşitlik 21'de verilen yaklaşıklanmış yeterli istatistikleri gösterir. Eşitlik 17'de verilen yardımcı fonksiyon güncellemesini yapabilmek için gerekli olan fonksiyon tanımları Eşitlik 24a-24f'de verilmiştir.

$$
\begin{aligned}
& s^{\mu}\left(z_{k}, z_{k-1}, y_{k}\right)=\mathbb{I}\left(r_{k}=j\right) \bar{s}^{\mu}\left(x_{k}, r_{k}=j, y_{k}\right) \\
& s^{X}\left(z_{k}, z_{k-1}, y_{k}\right)=\mathbb{I}\left(r_{k}=j\right) \bar{s}^{X}\left(x_{k}, r_{k}=j, y_{k}\right) \\
& s^{\pi}\left(z_{k}, z_{k-1}, y_{k}\right)=\bar{s}^{\pi}\left(x_{k}, r_{k}=j, y_{k}\right) \\
& \bar{s}^{\mu}\left(x_{k}, r_{k}=j, y_{k}\right)=\left[y_{k}-h\left(x_{k}\right)\right] \\
& \bar{s}^{X}\left(x_{k}, r_{k}=j, y_{k}\right)=\left[y_{k}-h\left(x_{k}\right)\right]\left[y_{k}-h\left(x_{k}\right)\right]^{T} \\
& \bar{s}^{\pi}\left(x_{k}, r_{k}=j, y_{k}\right)=\mathbb{I}\left(r_{k}=j\right)
\end{aligned}
$$

$\mathrm{Bu}$ eşitliklerde $\mathbb{I}($.$) gösterge fonksiyonudur. Bu$ fonksiyon, içindeki eşitlik sağlandığında 1, diğer durumlarda 0 değerini alır. Bu fonksiyon sayesinde istatistikler her bir alt-objenin bilinmeyen parametreleri için ayrı ayrı toplanır. Bu istatistikler basitçe, $\left[y_{k}-h\left(x_{k}\right)\right]$ ve $\left[y_{k}-h\left(x_{k}\right)\right]\left[y_{k}-h\left(x_{k}\right)\right]^{T}$ değerlerinin her bir alt-obje için ayrı ayrı toplanmasına karşılık gelir. 


\section{BENZETIMM SONUÇLARI}

$\mathrm{Bu}$ bölümde algoritmanın performansı bir simülasyon senaryosunda test edilmiştir. Simülasyonda 3 elips ile temsil edilen bir hedef yaklaşık olarak sabit hızla hareket etmekte ve her bir anda yüzeyi üzerinde ortalama değeri 9 olan Poisson dağılımlı ölçümler vermektedir. $\mathrm{Bu}$ hedefin sistem dinamikleri aşağıda verilen eşitliklerle ifade edilebilir [1],

$x_{k}=F x_{k-1}+e_{k}, \quad y_{k}^{j}=H x_{k}+w_{k}\left(r_{k}^{j}\right)$,

$E\left\{e_{k} e_{k}^{T}\right\}=\left[\begin{array}{cc}\frac{T^{3}}{3} I_{2} & \frac{T^{2}}{2} I_{2} \\ \frac{T^{2}}{2} I_{2} & T I_{2}\end{array}\right], F=\left[\begin{array}{cc}I_{2} & T I_{2} \\ 0_{2} & I_{2}\end{array}\right]$,

$\mathrm{H}=\left[\begin{array}{ll}\mathrm{I}_{2} & 0_{2}\end{array}\right]$

Bu eşitliklerde $I_{2}$ ve $0_{2}, 2 \times 2$ 'lik özdeşlik ve sıfır matrislerini; $\quad x_{k}, \quad$ hedefin Kartezyen koordinatlarındaki pozisyon ve hızını içeren durum vektörünü $\quad\left(x_{k}=\left[p_{k}^{x} p_{k}^{y} v_{k}^{x} v_{k}^{y}\right]^{T}\right) ; T$ değişkeni ise örnekleme zamanını gösterir. Simülasyonda $T=1$ sn olarak kabul edilmiştir. Simülasyon uzunluğu gösterim kolaylığı için 10sn alınmıştır. Simülasyonlarda algoritma 1000 parçacık kullanılarak çalıştırılmıştır. Hedef şeklini tanımlayan elipslerin merkezleri ve kovaryansları aşağıdaki gibi seçilmiştir.

$$
\begin{aligned}
& \mu_{1}=\left[\begin{array}{l}
0 \\
0
\end{array}\right], \mu_{2}=\left[\begin{array}{l}
0 \\
-5
\end{array}\right], \mu_{3}=\left[\begin{array}{l}
0 \\
5
\end{array}\right], \\
& \mathrm{R}_{1}=\left[\begin{array}{cc}
50 & 0 \\
0 & 2
\end{array}\right], \mathrm{R}_{2}=\left[\begin{array}{cc}
8 & 4,5 \\
4,5 & 5
\end{array}\right], \mathrm{R}_{3}=\left[\begin{array}{cc}
8 & -4,5 \\
-4,5 & 5
\end{array}\right]
\end{aligned}
$$

Şekil 2'de hedefin şekli ve pozisyonunun, gerçek (turuncu-kesikli) ve kestirilen (mavi-sürekli) değerleri gösterilmiştir. Şeklin en sol tarafına tekabül eden başlangıç noktasında, hedefin şekli henüz bilinmemektedir. Bu anda var sayılan hedef, Şekil 3'te gösterilmiştir. Zaman içerisinde toplanan ölçümlerle kestirilen şekil, gerçek hedef şekline başarıyla yakınsamıştır. Son andaki kestirim sonucu Şekil 4'te gösterilmiştir. Algoritma çıktısında hedef şeklini tanımlayan elipslerin merkezlerinin kestirilen değerleri, kovaryansların kestirilen değerleri ile birlikte aşağıda verilmiştir. Bulunan değerler, Şekil 4'te de gözlemlenebileceği üzere, gerçek değerlere oldukça yakındır.

$$
\begin{aligned}
& \widehat{\mu_{1}}=\left[\begin{array}{l}
1,08 \\
0,36
\end{array}\right], \widehat{\mu_{2}}=\left[\begin{array}{c}
0,4 \\
-4,6
\end{array}\right], \widehat{\mu_{3}}=\left[\begin{array}{l}
0,63 \\
5,33
\end{array}\right], \\
& \widehat{X_{1}}=\left[\begin{array}{cc}
56,03 & -1,04 \\
-1,04 & 1,5
\end{array}\right], \widehat{X_{2}}=\left[\begin{array}{ll}
7,12 & 4,26 \\
4,26 & 4,76
\end{array}\right], \\
& \widehat{X_{3}}=\left[\begin{array}{cc}
6.81 & -4.45 \\
-4.45 & 5.75
\end{array}\right]
\end{aligned}
$$

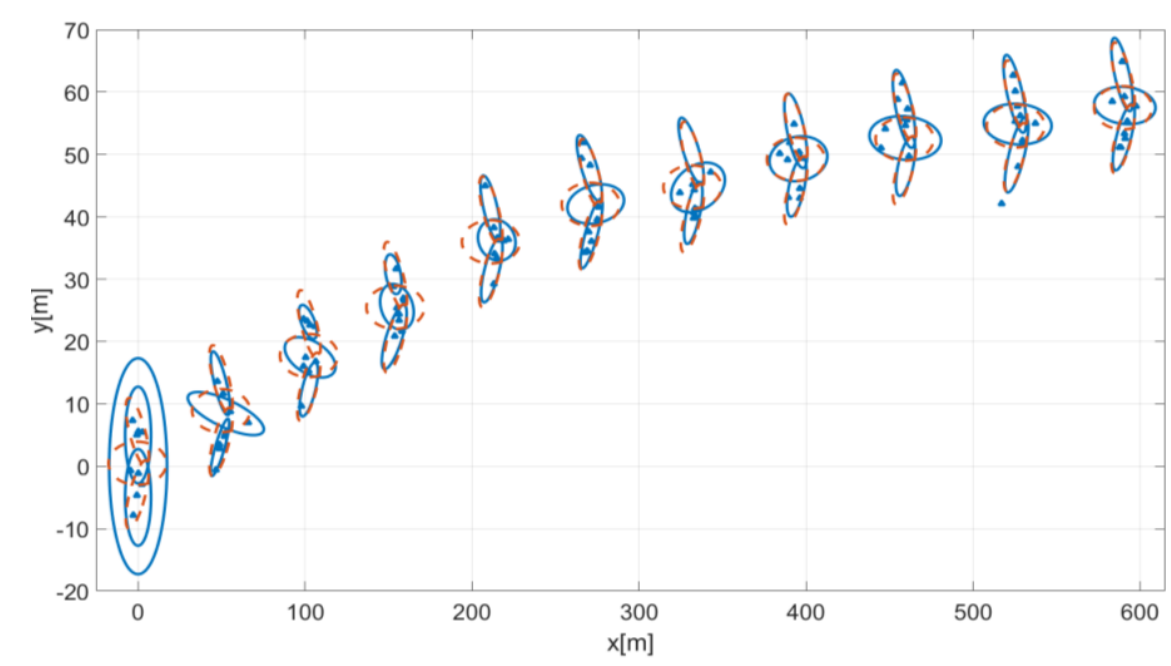

Şekil 2. Test senaryosu sonuçları 


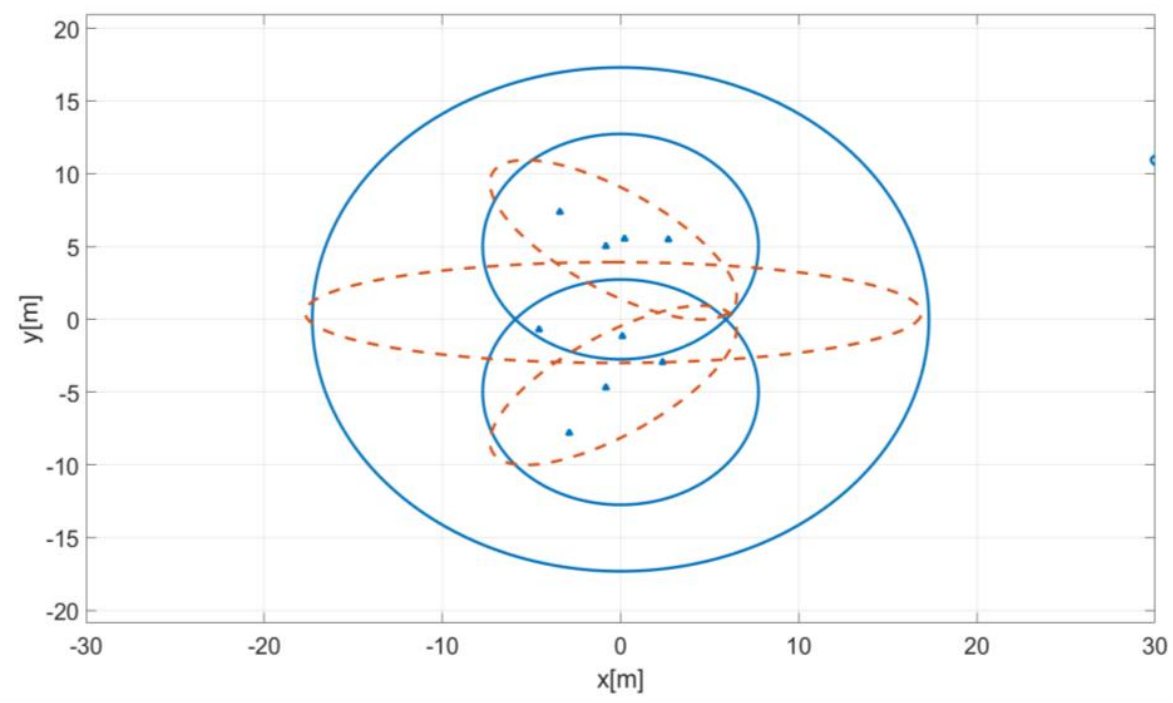

Şekil 3. Senaryo başlangıç anındaki gerçek (turuncu-kesikli) ve tahmini (mavi-sürekli) hedef şekli

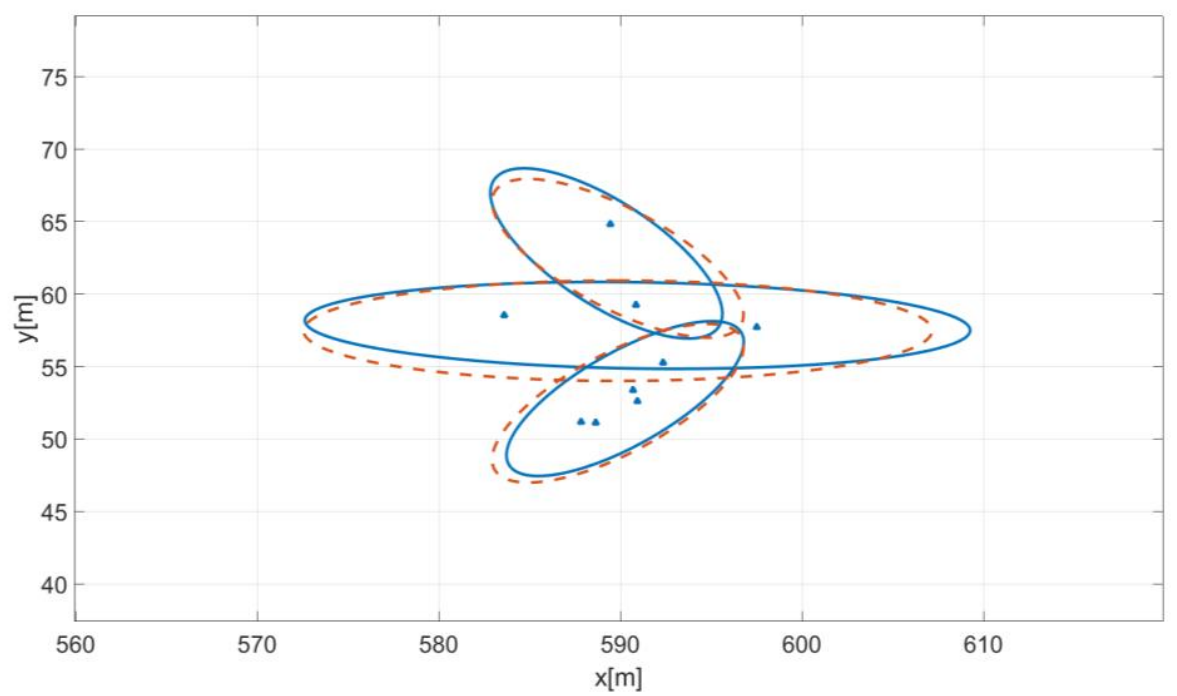

Şekil 4. Senaryo bitiş anındaki gerçek (turuncu-kesikli) ve tahmini (mavi-sürekli) hedef şekli

\section{TARTIŞMA VE SONUÇ}

Bu çalışmada doğrusal olmayan ve kombinatorik zorluklar içeren bir GHİ problemi için BM çerçevesinde yeni bir çözüm metodu geliştirilmiştir. Önerilen yeni metot, diğer GHİ çözümlerinin aksine buluşsal yaklaşımlar içermemektedir. Yapılan simülasyonlarda, algoritmanın takip ve şekil kestirim başarısı sergilenmiştir.

\section{KAYNAKLAR}

1. Bar-Shalom, Y., Rong Li, X., Kirubarajan, T., 2004. Estimation with Applications to Tracking and Navigation: Theory Algorithms and Software, John Wiley \& Sons. 
2. Granström, K., Baum, M., Reuter, S., 2017. Extended object tracking: Introduction, Overview and Applications, ISIF Journal of Advances in Information Fusion, 12(2), 139-174.

3. Koch, J.W., 2008. Bayesian Approach to Extended Object and Cluster Tracking Using Random Matrices, IEEE Transactions on Aerospace and Electronic Systems, 44(3), 104-1059.

4. Feldmann, M., Franken, D., Koch, W., 2011. Tracking of Extended Objects and Group Targets Using Random Matrices, IEEE Transactions on Signal Processing, 59(4), 1409-1420.

5. Dempster, A.P., Laird, N.M., Rubin, D.B., 1977. Maximum Likelihood from Incomplete Data Via the EM Algorithm, Journal of the Royal Statistical Society. Series B (Methodological), 39(1), 1-38.

6. Moon, T.K., 1996. The ExpectationMaximization Algorithm, IEEE Signal Process. Mag., 13(6), 47-60.

7. McLachlan, G.J., Krishnan, T., 1997. The EM Algorithm, Extensions ser. Wiley Series in Probability and Statistics, NY USA: John Wiley \& Sons Inc.

8. Cappé, O., Moulines, E., Rydén, T., 2005. Inference in Hidden Markov Models, Springer Series in Statistics, New York NY USA: Springer Science + Business Media LLC.

9. Schön, T.B., Wills, A., Ninness, B., 2011. System Identification of Nonlinear State-space Models, Automatica, 47(1), 39-49.

10. Cappé, O., 2011. Online EM Algorithm for Hidden Markov Models, Journal of Computational and Graphical Statistics, 20(3), 728-749.

11. Cappé, O., 2009. Online Sequential Monte Carlo EM Algorithm, Statistical Signal Processing SSP '09. IEEE/SP $15^{\text {th }}$ Workshop on, Cardiff, Wales, 37-40.

12. Doucet, A., de Freitas, N., Gordon, N., 2001. Sequential Monte Carlo Methods in Practice, NY, USA: Springer-Verlag.

13. Bishop, C.M., 2007. Pattern Recognition and Machine Learning, Springer. 